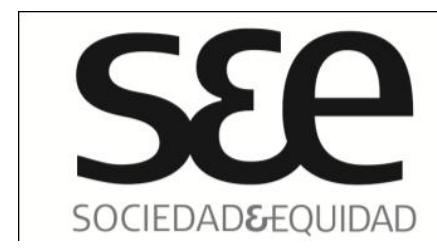

\title{
Educación y desigualdad en Chile: avanzando hacia espacios interculturales
}

Education and Inequality in Chile: moving towards intercultural spaces

\begin{tabular}{ll}
\hline & \\
Nombre: & Marcela Amaya García (1) \\
Filiación: & Universidad Católica Silva \\
& Henríquez \\
País: & Chile \\
Correo: & mamaya@ucsh.cl
\end{tabular}

\section{Resumen}

En el presente ensayo se reflexiona sobre el reto de resignificar los espacios de aula en el contexto educacional chileno, atendiendo a las tensiones y dilemas propios de un sistema que ha estado cruzado por la razón instrumental, el universalismo y el liberalismo político-económico en tanto sustratos epistémicos, lo cual ha devenido en importantes brechas de estratificación entre los estudiantes. De este modo, a partir de la descripción de la situación del microcosmos educativo y de la revisión del estado del arte de la interculturalidad en Chile, se propone como un horizonte posible, tendiente a soslayar las desigualdades sociales del sistema educacional, el avance hacia la implementación de políticas educacionales de índole intercultural, donde cobre realce el rol del Estado como garante de iniciativas que promuevan entre los estudiantes el reconocimiento con y entre los otros; el desarrollo de habilidades cognitivas reflexivas sobre sus prácticas culturales; afectivas referidas a la capacidad de regular su propio comportamiento y comunicativas necesarias para una vida en comunidad, pues resulta central el fomento del rol ciudadano.

\footnotetext{
${ }^{1}$ La autora es Periodista, Magíster en Lingüística y Dra. (c) Ciencias de la Educación. Sus líneas de investigación son: educación intercultural, análisis crítico del discurso, lingüística aplicada y didáctica de la lengua.
} 


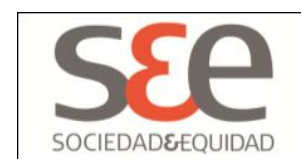

\title{
Palabras Claves
}

Educación, Interculturalidad, Desigualdad social, Alteridad, Sociedad global.

\begin{abstract}
This essay reflects on the challenge of re-signifying classroom spaces in the Chilean educational context, considering the tensions and dilemmas of a system that has been crossed by instrumental reason, universalism and liberalism as politicaleconomic epistemic substrates, which has resulted in important stratification gaps among students. Thus, from a description of the situation of the educational microcosms and a review of the state of the art regarding interculturality in Chile, we propose as a possible horizon, avoiding the social intercultural inequalities of the education system, the progress towards the implementation of an educational policy, where the State has a key role promoting recognition among students: developing reflexive cognitive skills regarding their cultural practices, affective skills regarding their capacity to regulate behavior, and communicative skills necessary for community life, central to the citizen role.
\end{abstract}

\section{Keywords}

Education, Intercultural, Social inequality, Otherness, Global society.

Se estima que la movilización estudiantil chilena es actualmente la punta del iceberg de una problemática cuyo meollo se encuentra en la sistemática violencia simbólica (Bordieu y Passeron, 2001) ejercida por el sistema educativo. Éste, de base universalista-liberal, reproduce la cultura de las clases dominantes, dejando con ello fuera el reconocimiento y respeto por la alteridad, o sea, la diversidad.

Al respecto, datos publicados en www.universitarios.cl, relativos al último estudio del Fondo de las Naciones Unidas para la Infancia (UNICEF) acerca de los resultados de la Prueba PISA son decidores: "Un 30\% de los jóvenes de clase acomodada tendrá durante toda su vida escolar solamente compañeros de su mismo nivel social". Una postura similar presenta la Organización para la Cooperación y el Desarrollo Económico, que el año 2004 afirmaba que la educación chilena está conscientemente estructurada en clases sociales (OCDE, 2004). 


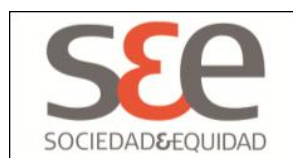

Por otro lado, Morín (2011) y Skiliar (2009) comentan que asistimos a una crisis: el primero referirá a una crisis planetaria producto de la globalización, mientras que el segundo a una crisis de la concepción de escuela.

Ante esto, se deben buscar vías para cambiar el colapsado modelo educacional chileno. Una opción es la educación intercultural como eje epistemológico, cuyo objetivo es resignificar los espacios educativos para siguiendo a Skiliar (2009)- estar-juntos en la educación.

En este ensayo se desarrollarán conceptos como el de razón instrumental, liberalismo político-económico y universalismo, en tanto antecedentes epistémicos del sistema educativo implementado en Chile. Luego se profundizará en las complejidades del concepto de interculturalidad y su estado del arte en nuestro país. Por último, se proponen ciertas directrices tentativas para la adscripción de una educación pública intercultural como horizonte posible. Desde luego, no se espera agotar las materias abordadas, sino más bien evidenciar un camino de debates necesarios y emergentes.

\section{Razón instrumental, universalismo y liberalismo político-económico como paradigma educativo}

Si se analizan los planes y programas elaborados por el Ministerio de Educación, correspondientes a los subsectores fundamentales, esto es, Lenguaje y Matemáticas, se constata que presentan una marcada inclinación hacia una mirada técnica, basada en el cumplimiento de objetivos y metas, cuyo origen estaría en "el advenimiento de la educación de masas, (...) relacionado con la necesidad sentida por el moderno estado industrial de disponer de mano de obra adiestrada (...)" (Kemmins, 1998: 48).

Aún más, se debe precisar que dichas concepciones cruzan el proceso educativo, ya que, considerando a Warr (1996), los profesores no pueden desligarse de sus visiones teóricas ni creencias. Ello lo ratifica Contreras (2010:1) al señalar que “(...) al considerar el conjunto de las creencias y la relación entre lo que se debe y creen hacer, los resultados indican que los profesores se sitúan entre dos teorías sobre el aprendizaje cercanas a un modelo tradicional: la teoría directa y la interpretativa".

Desde luego, el desarrollo epistemológico desde Descartes hasta Marx ha sido de tipo eurocentrista, es decir, ha tenido, por una parte, una impronta que niega la subjetividad del sujeto de la enunciación en función de la epistemología de la neutralidad y la objetividad empírica del sujeto que produce conocimiento científico y, por otra, el arraigo de un universalismo de la tradición filosófica en que dicho conocimiento es eterno, impermeable al tiempo y al espacio. ¿Qué ha significado esto? En pocas palabras, ha implicado que las ciencias occidentales, y 


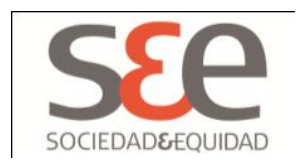

entre ellas las Ciencias de la Educación, han girado bajo el universal abstracto de Occidente, que ha impuesto lógicas verticales de poder, de orden entre los pueblos, borrando las diversidades, excluyendo a los otros y perpetuando el colonialismo e imperialismo.

Al respecto, Femenías (2007) explica que el marco teórico de los debates contemporáneos y específicos, relativos al fenómeno de la multiculturalidad, se encuentran en la Ilustración. Es clave aquí Kant y sus aportes teóricos acerca de la teoría ético-política, que promovía en el hombre la “(...) libertad de hacer uso público de su razón íntegramente" (Kant, 2009: 250), es decir, llamaba a la toma de conciencia de su propia libertad y, por tanto, de su autonomía. Sobre este punto, Güell (2008: 41) hace un aporte importante, que nos permite comprender el planteamiento kantiano: “El individuo, en un acto de pura reflexión, podría identificar de manera 'clara y distinta' los principios de una razón que, a modo de una geometría, podían dar certeza al conocimiento y racionalidad al ordenamiento del mundo".

Se debe señalar que para Kant la llustración es la libertad de hacer siempre y, en todo lugar, un uso público de la razón. De este modo, como dice Güell (2008: 41), la promesa ilustrada se soportaba en la idea de la "existencia de una identidad entre la razón, cuyos principios descubría el intelecto, y la razón que subyacía al orden de la naturaleza". Dicho de otro modo, todos los hombres podrían ser libres al pensar por su propia voluntad, pues el uso público de su razón - de acuerdo a Kant- debía estar permitido sin excepciones. Por lo tanto, la razón ayudaría a combatir la ignorancia, la superstición y la tiranía y construir un mundo mejor.

Sin dudas, esto último abrió la puerta para el establecimiento de pautas universales de comportamiento del hombre, donde la base era la igualdad entre los individuos, ya que expresaba la relación entre el individuo y la razón pública. Según Femenías (2007: 53) “(...) el universal constituye el criterio que (...) sentó las bases teóricas para desmontar el edificio del orden jerárquico natural que daba a cada cual un lugar natural diferenciado" y se ha constituido en pilar del pensamiento liberal o liberalismo. En efecto, este sistema filosófico, político y económico, por una parte, aboga por el desarrollo de las libertades individuales y, por otra, establece un Estado de Derecho que reconoce la igualdad de las personas ante la ley, sin privilegios ni distinciones, mediante la Constitución Política que sirve de marco mínimo regulatorio de las libertades de las personas. En palabras de Rawls (1993: 35), el liberalismo político en tanto concepción política apunta a lo siguiente: “(...) Hacia una concepción política de la justicia como punto de vista libremente aceptado. Uno de los objetivos es (...) delimitar el dominio político y especificar su concepción de la justicia de tal manera que sus instituciones puedan obtener el apoyo de un consenso traslapado". 


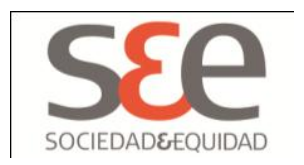

De la cita anterior, entonces, se desprende que en el liberalismo políticoeconómico se instala el principio de la "sociedad como sistema justo de cooperación social entre personas libres e iguales" (Rawls, 1993: 34). Por lo tanto, aquí es central la concepción política de la persona, pues "los ciudadanos son libres en la medida en que se conciben a sí mismo y unos a otros como poseedores de la capacidad moral para tener una concepción del bien" (Rawls, 1993: 51). Por cierto, según el autor las sociedades democráticas se sustentan en dos principios de justicia, definida ésta como: “(La) capacidad moral que tenemos para juzgar cosas como justas, apoyar esos juicios en razones, actuar de acuerdo con ellos y desear que otros actúen de igual modo. Sin embargo este proceso se da a nivel de los individuos en el marco de la sociedad y su estructura básica" (Caballero, 2006: 5).

Así, los principios son: será justa aquella sociedad que aporta la mayor libertad posible a los individuos, donde ésta sea compatible con la mayor libertad posible del otro y las desigualidades sociales sean aceptadas a condición de que generen beneficios a los menos aventajados de la sociedad (principio de la diferencia) y los cargos y puestos deben de estar abiertos para todos bajo condiciones de igualdad de oportunidades (justa igualdad de oportunidades). Según Caballero (2006), con este último principio, Rawls pretende dar una alternativa al sistema de libertad natural.

Para Taylor (1993), lo anterior resulta ser un desafío endémico en las democracias liberales, ya que por principio están comprometidas con la igualdad de representación para todos y no con el reconocimiento de las diferencias. Incluso, Gutmann (en Taylor, 1993: s/n) reconoce que "las instituciones, incluyendo las dependencias del gobierno; las escuelas y los colegios y universidades de artes liberales, han sido el blanco de severas críticas en estos días por no reconocer la identidad cultural particular de los ciudadanos". Zizek (2009) va más allá, diciendo que la categoría de multiculturalidad liberal es la ideología del capitalismo mundializante, de la era posmoderna, del post Estado-Nación que predica la tolerancia entre culturas, porque respeta la identidad del "otro", pero como una forma de demostrar y plasmar su propia superioridad. Desde luego, en los dichos del autor anterior subyace la idea de liberalismo económico, que despojó a las sociedades democráticas liberales de las regulaciones económicas del absolutismo, permitiendo el desarrollo de la economía del mercado y el reinado, por ende, del capitalismo, caracterizado por ser un orden social donde se privilegia el usufructo de la propiedad privada sobre el capital como herramienta de producción y las Tecnologías de la Información (TIC's) encuentran su máxima expresión como dinamizadoras de atracción de otras tecnologías, contribuyendo así a la productividad del resto de los sectores empresariales en base a su horizontalidad (Revilla, 1991). 


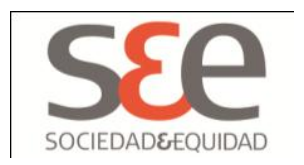

En la globalización, entendida por McLuhan y Powers (1995) como el marco de la interconectividad a nivel mundial, que implica estrechez de vínculos económicos, políticos y sociales entre las naciones producto de la irrupción de las TIC's. Dice Zizek (2009) que sólo tiene lugar una "universalidad concreta" que da espacio a la existencia de un orden mundial, donde el capitalismo global a través del mercado promueve la tolerancia, respeto y protección a la Democracia. En síntesis, para el autor la libre elección y la libertad de la promesa kantiana funcionan como un mero gesto formal, ya que el sujeto de libre elección solamente puede emerger como resultado de un extremadamente violento proceso de desvinculación de su mundo vital, al desprenderse de sus raíces particulares culturales/sociales, para afirmar su plena autonomía y su universalidad. Este tipo de hombre, podría ser asimilable al que ha sido denominado por Rojas (2003) como "hombre light", quien se caracteriza por ser un individuo sin sustancia, hedonista, que no corre riesgos, consumista, superfluo, alejado del desarrollo de ideas y cuestionamientos, sin ideales, relativista, subsumido en su propio yoísmo. Habermas (1999: 102), finalmente, reforzando lo expuesto por Zizek (1998), señala que “(...) con el fin del Estado Nacional también cortamos simultáneamente con el proyecto de la autonomía ciudadana (...)", por lo tanto, en el mundo postpolítico “(...) los ciudadanos serán arrojados a un mundo de redes anónimas en el que tendrán que decidir según propias preferencias entre opciones creadas en términos sistémicos".

\section{Microcosmos educativos estratificados}

Según Vidal (2006), si bien el sistema educacional chileno ha avanzado en la cobertura, persisten las inequidades en torno a las oportunidades de aprendizaje y años de escolarización. De acuerdo a la autora, esto se debe a la constante influencia que ha sufrido la educación por parte del neoliberalismo económico.

Estas inequidades se derivan básicamente de un modelo económico de alta concentración del ingreso, que genera una fuerte estratificación social y educacional, a lo cual se agregan pautas culturales muy arraigadas, relacionadas con la discriminación y el no respeto por la diferencia (Vidal, 2006: 139). También, esta autora indica que "la estratificación del sistema educacional tiende a reproducir la estratificación social y a su vez el origen social es un factor decisivo del niño o la niña en el sistema escolar" (Vidal, 2006: 139). A la vez, identifica dos grandes focos de desigualdad: de resultados en el colegio (el factor familia) y de resultados finales (educación superior y mundo laboral).

Bajo esta lógica, Vidal (2006: 139) asevera que una primera medida para terminar con la desigualdad social en Chile dice relación con "la necesidad de contar con políticas educacionales eficaces y eficientes”, así como implementar políticas 


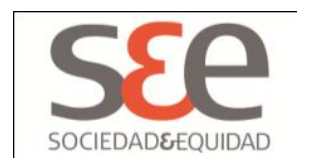

redistributivas de los ingresos. Por lo tanto, centra el debate en el papel del Estado como garante de la educación y hace un llamado a resolver estas problemáticas, poniendo hincapié en la inversión de recursos.

En cualquier caso, marcando un distanciamiento con la investigadora antes citada, aquí el eje en discusión no tiene que ver, necesariamente, con una cuestión financiera, sino más bien con el tipo de representación social de la escuela y la educación deseable de instalar para que sea posible la tríada: Educación, Desigualdad e Interculturalidad.

Sobre este punto, Quilaqueo, Quintriqueo y Cárdenas (2005: 208, citando a Quintriqueo \& Maheux, 2004) señalan que la "relación entre la realidad sociocultural, por una parte, y la representación de la escuela y de la educación, de otra parte, están ligadas a una concepción que cada sociedad tiene respecto de la formación de la persona". Por ello, Quilaqueo, Quintriqueo y Cárdenas (2005: 252), consideran que "la implementación de una educación intercultural, sigue presentando una serie de desafíos". Sobre esto, Contreras (2011) explica que los grandes obstáculos a salvar para dar lugar a una educación de este tipo están dados por, justamente, la estructura segmentada del sistema educacional chileno, que no permite la relación de grupos sociales distintos y el abordaje como debate de los conceptos de Nación y alteridad en tanto ejes de una política intercultural. En forma concomitante, como otro escollo difícil de roer en relación a su alto impacto en el imaginario nacional, está el uso sinonímico y, desde luego, equívoco a nivel de la comunidad de habla y de las autoridades chilenas de los conceptos de interculturalidad y multiculturalidad. El primero designa "la tríada diversidadsingularidad y universalidad" (Saéz, 2008: 121) y, el segundo, "supone aceptación de lo heterogéneo (García Canclini, 2004: 15). De ahí, que bajo las miradas multiculturalistas, como la presente en Chile, se admita la diversidad de culturas, recalcando las diferencias de sus miembros y activando políticas compensatorias de respeto.

\section{Interculturalidad: Un concepto en construcción}

Cuando se habla de interculturalidad, lo primero que se debe reconocer es que no existe una definición unívoca y que el debate conceptual está lejos de terminar, dándose -a nuestro juicio- en la actualidad un verdadero carnaval de perspectivas y aproximaciones propias de un término eminentemente polisémico.

Según Dietz (2001), el concepto de estudios interculturales ha sido acuñado para designar un campo emergente de preocupaciones transdisciplinarias en torno a los contactos y las relaciones que a nivel individual como colectivo se articulan en contextos de diversidad y heterogeneidad cultural. En el fondo, el autor pone 


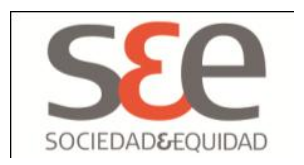

en el tapete la emergencia de los trabajos en el área, lo que se condice con los bordes difusos con que cuenta.

Por otra parte, una mirada -creemos- más radical, decidora y factible de asir, es la que presenta Schmelkes (2002), sosteniendo que la interculturalidad implica la toma de una postura política que debe promover el cambio de las relaciones de asimetría que caracterizan la sociedad homogeneizadora. En este caso, la interculturalidad implica una relación respetuosa entre culturas, donde se pone énfasis en la superación de tres tipos de asimetrías: cultural, social y educativa. Entonces, a nuestro juicio, esta última será clave para la construcción de un país intercultural, ya que esto no puede ocurrir sin una actividad educativa de esa índole con toda la población de un país.

Una postura similar, en tanto encuentro de los miembros de la sociedad, pero menos empoderada de un posicionamiento, es la que presenta García Canclini (2004), señalando que la interculturalidad remite a la confrontación y el entrelazamiento, a lo que sucede cuando los grupos entran en relaciones de intercambios. De este modo, este teórico aboga porque la interculturalidad se transforme en un centro de comprensión y articulación, donde se garantice el ejercicio de la diferencia en espacios urbanos, en las migraciones nacionales e internacionales, en el reconocimiento universal de derechos. Es decir, esta perspectiva tiene una fuerte base en el pensamiento universalista como los Derechos Humanos.

Briones (2008), por su parte, releva como un elemento crucial la tensión respecto a los conceptos de diversidad e interculturalidad, por cuanto constituyen propuestas diferentes que, en general, se emplean indistintamente. Por ello, subraya que la interculturalidad opera como horizonte de significación de posibilidades, y es apto para promover ciudadanías activas más emancipadoras. De esta definición se desprende que el consenso e implícitamente el diálogo serían caminos posibles para promover espacios sociales de interculturalidad. Sin embargo, una crítica que podríamos hacer a esta reflexión dice relación con que no profundiza en cómo aquello se articula, quedando, quizás, en deuda.

Asimismo, interesa realzar el aporte de Besalú (2010), quien recalca que la interculturalidad no puede ser un barniz superficial que recubre y oculta lo ya existente, ni un añadido cortés y voluntarioso a una programación ya de sí sobrecargada, sino un poderoso foco que ilumine lo que acontece en la escuela.

Ahora bien, la Organización de Naciones Unidas para la Educación, la Ciencia y la Cultura, presenta el debate teórico sobre educación y multiculturalismo en el texto Directrices de la UNESCO sobre la Educación Intercultural (UNESCO, 2006). Aquí se desarrollan los principales aspectos e interrelaciones, dando cuenta, aunque de manera muy breve y sistémica, de las complejidades que considera la aproximación a esta racionalidad teorética. Sobre 


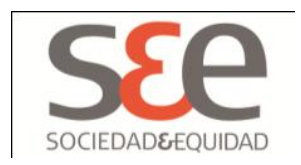

todo, la discusión teórica presente en el discurso de la UNESCO como escenario, consideramos que pone en el tapete un asunto latente en función de estas cuestiones, esto es, la vinculación del multiculturalismo con problemáticas de las culturas minoritarias. Dentro de ellas es posible identificar los pueblos autóctonos, las minorías territoriales y no territoriales y los inmigrantes. Es más, se puntualiza que la interculturalidad es un concepto mucho más dinámico y más amplio.

Siguiendo con esta línea de reflexión, es menester rescatar el enfoque de educación intercultural planteado por la UNESCO, donde el diálogo, el conocimiento mutuo y el respeto son los ejes para la convivencia evolutiva y sostenible en las sociedades multiculturales, por cierto, no interculturales. Aquí, la pregunta que deberíamos hacernos es: ¿Por qué este trazado?, ¿a qué tipo de convivencia se hace referencia? La respuesta a la primera interrogante consideramos que resulta casi obvia: la educación, según la UNESCO, tiene una misión importante que cumplir en la promoción de la cohesión social y la coexistencia pacífica. La contestación a la segunda pregunta también creemos que es lógica, ya que se trata de una declaración de intenciones que implicaría el consenso instantáneo.

De esta forma, si se hace un resumen de lo expuesto hasta ahora, se tiene que las distintas aproximaciones de interculturalidad reivindican al interior del concepto al sujeto y el rescate de su diversidad, cuestión que se entiende como un avance en el reconocimiento de las interrelaciones.

\section{Educación intercultural en Chile: Estado del arte}

Para comenzar, se debe señalar que en Chile la legislación relativa a los temas interculturales es exigua, destacándose el resguardo de los Derechos Humanos en forma explícita en la Constitución Política de 1981 en Artículo 1. Particularmente, las problemáticas referidas a la diversidad en sus distintas manifestaciones han sido tratadas, por una parte, en la Ley 19. 253 de 1993, que aborda la temática de los pueblos indígenas y, por otra, en la Ley 23.592, también conocida como Ley de Antidiscriminación, donde se vela por el respeto a la diversidad.

Una situación similar acontece respecto de las políticas del Ministerio de Educación en relación a la promoción de una educación intercultural en el país, siendo la principal iniciativa de este tipo el Programa de Educación Intercultural Bilingüe, que “(...) surgió en Chile porque existía la necesidad de complementar y modificar una serie de aspectos que la educación estándar no estaba proporcionando" (Raidemann, 2008: 170). 


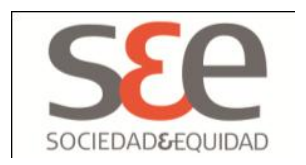

El Programa de Educación Intercultural Bilingüe se orienta a la incorporación de textos bilingües, distribución de softwares de las culturas y lenguas indígenas del país en escuelas y liceos, creación de sistemas de inmersión de lengua Rapa Nui en la Isla de Pascua, contextualización de programas de estudios a la realidad cultural y lingüística de los estudiantes, desarrollo de proyectos educativos institucionales con participación de las comunidades indígenas, participación de autoridades indígenas en actividades pedagógicas de la escuela y generación de modelos de uso de nuevas tecnologías en escuelas con población indígena. En estos momentos, el Programa se encuentra en su segunda fase de aplicación, trabajando con 78 establecimientos a nivel nacional. Por lo tanto, puede decirse que se trata de una instancia limitada y con carácter más bien remedial, por cuanto se centra solamente en la recuperación de las lenguas vernáculas.

A partir de lo antes expuesto, es posible afirmar que en Chile, la interculturalidad resulta ser un campo de acción constantemente descuidado a nivel del Estado. De ahí que se entiende que estamos asistiendo a lo que Skliar (2009) concibe como pérdida del sentido fundante y decisivo de la escuela, esto es, el educar a todos. Vale decir, el concepto de educación ya no daría cuenta de las demandas del mundo actual, mostrando una incapacidad para transmitir valores y virtudes a los enseñantes. En efecto, el nuevo movimiento social de los estudiantes, conceptualmente entendido bajo la perspectiva de Kymlicka (1996), se cree que es una muestra de la crisis educativa del sistema chileno, cuyo funcionamiento y regulación ha sido entregado por el Estado a las fuerzas del mercado, provocando, entre otros, la baja calidad de la enseñanza, el aumento explosivo de la matrícula y el endeudamiento de las clases sociales deprivadas para costear los estudios de sus hijos.

Pero podría establecerse que el tema es muchísimo más profundo y dice relación con la crisis de la convivencia a la que alude Skliar (2009) graficada a través una señal insoslayable, esto es, es la pérdida de la conversación como elemento de sentido de los procesos pedagógicos. Parece interesante comentar este aporte del autor antes mencionado, ya que -desde el punto de vista lingüístico-, la conversación es vista como un tipo de texto de menor jerarquía, puesto en perspectiva del diálogo. No obstante, para efectos de esta cavilación, la diferencia no sólo es semántica-conceptual, sino que se refiere a la concepción que tenemos del uso indistinto y equivocado de uno y otro término. Entonces, algunas aclaraciones. El diálogo corresponde a una tipología textual, donde es posible identificar una estructura prototípica compuesta por secuencias de apertura, intercambio y cierre, extrapolable a otros textos de iguales características, o sea, en el diálogo se establece una interacción comunicativa planificada y lejos de la espontaneidad. Por el contrario, en la conversación se 


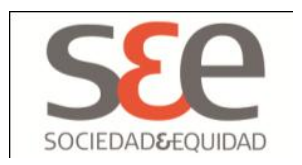

expresa plenamente la intención comunicativa de emisor y receptor, dando lugar a la retroalimentación, a los traslapes, los cambios de turno y a las subjetividades propias de las situaciones comunicativas poco estructuradas. Entonces, ¿por qué rescatar la conversación en el contexto de la educación? Porque a través de ella sería posible ver más allá de la opacidad de los discursos disciplinares pedagógicos que totalizan realidades y homogenizan conciencias.

En consecuencia, es fundamental cuestionar la concepción que la sociedad chilena tiene respecto de la formación de las personas, la que tiene una marcada inclinación hacia una mirada técnica basada en el cumplimiento de objetivos y metas, cuestión -que de acuerdo a Kemmins (1998)- estaría originada por el advenimiento de la educación de masas y la necesidad del estado moderno industrial de disponer de mano de obra adiestrada.

\section{Hacia una implementación de la resignificación: Algunas directrices tentativas}

En primer lugar, se debe señalar que como base de un proceso de resignificación de los espacios educativos, resulta imperioso revalorar el rol de la educación pública y, por ende, del Estado en la conformación de las futuras generaciones, puesto que "la misión de la escuela pública ha sido crear un grupo que comparta valores comunes, por encima de sus particularidades. Por tanto, lo que hace pública a esta escuela, no es sólo el servicio al público (que pueden prestarlo centros privados), sino 'crear' público o ciudadanos (Bolívar, 2004: 22).

Profundizando la idea anterior, el autor sostiene que "el objetivo de la escuela pública de integrar a la ciudadanía en unos principios y valores tiene entonces- que ser actualmente reformulado, para compatibilizar dicho fin con el reconocimiento de las diferencias de cada grupo o con los contextos locales comunitarios" (Bolívar, 2004: 23). En este sentido, la educación pública por definición, asegura el autor, se perpetúa como un espacio intercultural.

Sin cultura pública común no hay educación para la ciudadanía y se esfuma el sentido mismo de escuela pública. El asunto es qué debe de constituir dicha "cultura", de forma que no niegue las identidades culturales primarias ni queden relegadas al espacio privado, pero tampoco que su reafirmación impida dicha cultura común (Bolívar, 2004: 23).

En segundo lugar, se considera que una política educacional intercultural, impulsada por el Estado chileno, tendría que incorporar cambios a nivel macro dentro del currículum en sus tres dimensiones: currículum formal, currículum real y currículum oculto (Quilaqueo, Quintriqueo y Cárdenas, 2005, siguiendo a Perrenound, 1994). De esta manera, se impactarían los contenidos educativos; el 


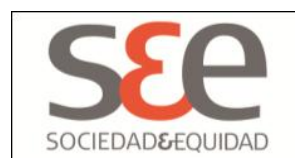

conjunto de experiencias orientadas hacia los aprendizajes relacionados, por cierto, con el tipo de sociedad a potenciar y las prácticas de enseñanza de los profesores.

Específicamente en cuanto al contenido curricular, una propuesta de educación pública intercultural debería abordar el aprendizaje situado y significativo, ya que empoderaría a los estudiantes en el reconocimiento de su propia realidad espacio-temporal, con el objetivo de gatillar la comprensión de los procesos políticos, económicos y sociales en los que viven inmersos.

En tercer lugar, la naturaleza epistemológica de una teoría de la educación transformadora se tendría que engarzar en un enfoque crítico (Warr, 1996), pues éste promueve el conocimiento de los propios sujetos, es decir, instala al sujeto como centro. Asimismo, entregaría a los profesionales de la educación autonomía para reflexionar desde su historicidad y cultura. De esta manera, ofrecería una oportunidad para el debate de la educación intercultural, ya que desecha la afirmación determinista de la práctica educativa, permitiendo transformaciones surgidas de la metacognición de la práctica para emanciparse de las creencias irracionales, erróneas heredadas de la tradición y la ideología, en este caso, instauradas desde el currículum nacional y la formación inicial tecnicista. Es decir, este enfoque empoderaría al profesional de la educación en la decisión didáctica de promover en las aulas la interculturalidad, en búsqueda, por ejemplo, del respeto, la solidaridad, la colaboración. Para lograrlo, sería crucial la formación inicial en las universidades y la elaboración por parte de los Ministerios de Educación de los estándares de calidad de los egresados bajo esta lógica de pensamiento. En cuanto a los profesores en ejercicio, se considera que el desarrollo de asistencias técnicas y de programas de especialización, tomando la interculturalidad como base epistémica, constituirían instancias importantes para el trabajo de esta temática.

En cuarto lugar, íntimamente relacionado con las prácticas educativas, se plantea como imperioso rescatar el diálogo como eje de las unidades didácticas a emplear en el aula, pues "hablar de interculturalidad y diálogo implica, necesariamente, hablar de cultura y lenguaje" (Gualdieri y Vásquez, 2011: 37). 0 sea, como dicen las mismas autoras, poner en el tapete la "pedagogía del encuentro" con los otros y con nosotros.

Una pedagogía del encuentro intercultural exige un cambio de nuestra Lingüística. Se entiende por cultura lingüística al conjunto de comportamientos, suposiciones, formas culturales, prejuicios, creencias, actitudes, estereotipos, opiniones sobre el lenguaje en general y sobre las 


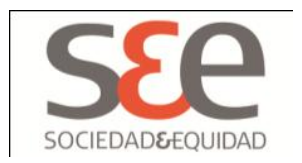

lenguas en particular (Gualdieri y Vásquez, 2011: 37, citando a Schiffman, 1998).

En quinto lugar, adentrándose en la didáctica y en consonancia con lo señalado, se estima que deberían reconsiderarse los modos de enseñar, atendiendo a las diferencias sociales y contextuales de los estudiantes y sus eventuales brechas de aprendizajes. Para ello, se cree imprescindible acoger en el aula la llamada didáctica no parametral, entendida como "una postura que comprende el proceso de enseñanza como un proceso intencional de permanente promoción de ruptura de sentidos y significados en el propio devenir de la existencia (...)" (Pineda y Hernando, 2007: 46, citando a Quintar, 2002). ¿Con qué objetivos se efectuarían estos ajustes didácticos? Las razones principales son las siguientes: desarrollar el pensamiento de los sujetos y potenciar en los estudiantes procesos de articulación para la construcción autónoma de nuevos conocimientos.

Al mismo tiempo, para fortalecer esta propuesta, se considera pertinente activar en las prácticas educativas intervenciones socioeducativas acordes a la interculturalidad, como es el caso del Aprendizaje Mediado impulsado por Feuerstein, quien empodera a los profesores como mediadores de significados, portadores de la función simbólica y de los estímulos ambientales (Kozulin, 2000). Aquí, entonces, cobraría especial relevancia la utilización de la evaluación dinámica en tanto “(...) herramienta de evaluación diagnóstica capaz de proveer pautas de intervención específicas para la instrumentación de una respuesta educativa más adecuada" (Sabina y Peralta, 2009: 123). Esta opción se entiende respondería a las complejidades propias de los estudiantes en diversidad educativa o deprivados.

Desde este mismo prisma, la interculturalidad en la educación pondría de relieve la pertenencia cultural de los sujetos y su relevancia social, es decir, el patrimonio cultural de los diferentes, poniendo en juego la idea de García Canclini (2004: 82) en cuanto a que “'el umbral de la ciudadanía' se conquista no sólo obteniendo respeto a las diferencias sino contando con los 'mínimos competitivos en relación con cada uno de los 'recursos capacitantes' para participar de la sociedad: trabajo, salud, poder de compra (...)".

Por último, se considera que el propio planteamiento epistemológico del concepto de interculturalidad, como bien lo hace notar Sáez (2008), abre la puerta hacia la puesta en práctica de la posibilidad de una educación intercultural pública, donde tenga cabida la convivencia de diversos sectores sociales y se tienda al reconocimiento de la alteridad de los otros, haciendo justicia así a las demandas de los estudiantes, en la actualidad, movilizados en búsqueda de una educación de calidad e igualitaria. 


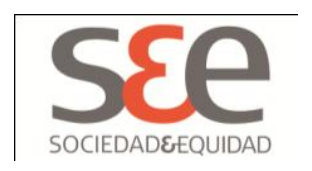

La interculturalidad es, sobre todo, un paradigma educativo emergente que apenas comienza a buscar caminos para su materialización y su concreción definiendo los aspectos y relaciones interculturales que generen mutuo entendimiento, o sea, el derecho de vivir la propia cultura, el intercambio y la comunicación enriquecedora donde cada individuo reconoce, acepta y trabaja la cultura del otro (Sáez, 2008: 108).

\section{Referencias Bibliográficas}

Besalú, X. (2010). La educación intercultural y el currículo escolar. Comunicación presentada en el I Congreso Internacional sobre Buenas Prácticas en Educación Intercultural, Málaga, España.

Bolívar, A. (2004). Ciudadanía y escuela pública en el contexto de diversidad cultural. Revista Mexicana de Investigación Educativa, núm. 20, Enero-Marzo 2004: 15-38.

Bordieu, P. y Passeron, J. C. (2001). La reproducción: Elementos para una teoría del sistema de enseñanza. España: Editorial Popular.

Briones, C. (2008). Diversidad cultural e interculturalidad: ¿De qué estamos hablando? En García, C. (Comp.), Hegemonía e interculturalidad: Poblaciones originarias y migrantes. La interculturalidad como uno de los desafíos del siglo XXI (pp.35-58). Buenos Aires: Prometeo.

Caballero, F. (2006). La teoría de la justicia de John Rawls. Ibero Forum, año I, núm. II, Otoño 2006: 1-22.

Contreras, S. (2010). Las creencias curriculares de los profesores de ciencias: Una aproximación a las teorías implícitas sobre el aprendizaje. Revista Horizontes Educacionales, núm. 15(1), 2010: 23 -36.

Contreras, D. (2011). Interculturalidad, infancia y políticas públicas. Manuscrito no publicado. 


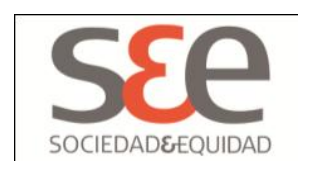

Dietz, G. (2001). Del multiculturalismo a la interculturalidad: Un movimiento social entre discurso disidente y praxis institucional. En de Prado, J. (Ed.), Diversidad cultural, identidad y ciudadanía (pp. 17-71). Córdoba: Instituto de Estudios Transnacionales.

Femenías, M. (2007). El género del multiculturalismo. Buenos Aires: U. de Quilmes.

García Canclini, N. (2004). Diferentes, iguales y desconectados. Mapas de la interculturalidad. Barcelona: Editorial Gedisa.

Güell, P. (2008). ¿Qué se dice cuando se dice cultura? Notas sobre el nombre de un problema. Revista de Sociología, año 22, 2004: 37-64.

Gualdieri, B. y Vásquez, M. J. (2011). El diálogo de la interculturalidad: Qué y cómo desde la práctica. En Loncon, E. y Hecht, A. (Comp.), Educación Intercultural Bilingüe en América Latina y el Caribe: Balances, desafíos y perspectivas (pp. 32-46). Santiago: Fundación Equitas y Programa de Doctorado en Ciencias de la Educación Mención Intercultural Universidad de Santiago.

Habermas, J. (1999). La inclusión del otro. Estudios de teoría política. Barcelona: Paidós.

Kant, I. (2009). ¿Qué es la ilustración? Foro de Educación, núm. 11, 2009: 249-254.

Kemmins, S. (1998). El currículum: Más allá de la teoría de la reproducción. Madrid: Ediciones Morata.

Kozulin, A. (2000). Instrumentos psicológicos. La educación desde una perspectiva sociocultural. Madrid: Paidós.

Kymlicka, W. (1996). Ciudadanía multicultural. Una teoría liberal de los derechos de las minorías. Barcelona: Paidós.

McLuhan, M. y Powels, B. R. (1995). La aldea global: Transformaciones en la vida y los medios de comunicación mundiales en el siglo XXI. Barcelona: Gedisa.

Morín, E. (2011). La vía. Para el futuro de la humanidad. Barcelona: Paidós. 


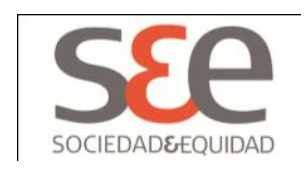

Organización para la Cooperación y el Desarrollo Económico (2004). Informe OCDE. París: Programa Regional Latinoamericano del Centro de la OCDE para la Cooperación de Países No Miembros (CCNM).

Pineda, A. y Hernando. L. (2007). Formación en tiempos presentes. Hacia pedagogías emergentes. Revista Latinoamericana de Estudios Educativos, núm. 1, Enero-Junio 2007: 41-63.

Quilaqueo, D.; Quintriqueo, S. y Cárdenas, P. (2005). Educación, currículum e Interculturalidad. Elementos sobre formación de profesores en contextos mapuches. Temuco: Universidad Católica de Temuco.

Raidemann, A. (2008). La educación intercultural bilingüe en Chile: ¿Ampliación de oportunidades para alumnos indígenas? Indiana, 25, 2008: 169-193.

Rawls, J. (1993). Liberalismo político. México D.F.: FCE.

Revilla, E. (1991). Reflexiones en torno al valor estratégico de la tecnología de la información. Anales de Estudios Económicos y Empresariales, núm. 6, 1991: 67-82.

Rojas, E. (2003). El hombre light: Una vida sin valores. Madrid: Temas de Hoy.

Sabina, A. y Peralta, O. (2009). Dificultades de aprendizaje. Evaluación dinámica como herramienta diagnóstica. Revista Internacional de Psicología y Educación, núm. 2, 2009: 113-139.

Sáez, R. (2008). La educación intercultural para una sociedad global. En García, C. (Comp.), Hegemonía e interculturalidad: Poblaciones originarias y migrantes La interculturalidad como uno de los desafíos del siglo XXI (pp. 103-129). Buenos Aires: Prometeo.

Schmelkes, S. (2002). La enseñanza de la lectura y la escritura en contextos multiculturales. Comunicación presentada en el VII Congreso Latinoamericano para el Desarrollo de la Lectura y la Escritura, Puebla, México.

Skliar, C. (2009). Educar a cualquiera y a cada uno. Sobre el estar-juntos en la educación Comunicación presentada en las VI Jornadas de Cooperación Educativa con Iberoamérica sobre Educación Especial e Inclusión Educativa. Estrategias para el Desarrollo de Escuelas y Aulas Inclusiva, Antigua, Guatemala. 


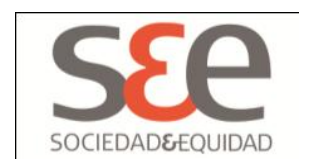

Taylor, Ch. (1993) El multiculturalismo y la política del reconocimiento. México D.F.: FCE.

Organización de las Naciones Unidas para la Educación, la Ciencia y la Cultura (2006). Directrices de la UNESCO para la educación intercultural. Francia: UNESCO.

Vidal, P. (2006). Educación y desigualdad. Revista de la Academia, núm. 11, Primavera 2006: 139-156.

Warr, W. (1996). Una teoría para la educación. Madrid: Ediciones Morata, S. L.

Zizek, S. (1998). Multiculturalismo o la lógica cultural del capitalismo multinacional. En Jameson, F. y Zizek, S. Estudios culturales. Reflexiones sobre el multiculturalismo (pp. 137-188). Buenos Aires: Paidós.

Zizek, S. (2009). Sobre la violencia. Seis reflexiones marginales. Buenos Aires: Paidós. 